\title{
djb-Modell für eine geschlechtergerechte Rentenreform
}

Ziel des djb-Modells ${ }^{1}$ ist eine maßvolle Umverteilung im System der gesetzlichen Rentenversicherung, das derzeit den Einkommensunterschied in der Zeit der Erwerbstätigkeit im Alter überproportional verstärkt. Das Modell sieht drei Änderungen vor:

1. In das Leistungsrecht der gesetzlichen Rentenversicherung wird ein Mindestsicherungselement, der Grundbetrag, eingebaut. Er bildet mit dem Steigerungsbetrag die individuelle Rente.

2. Die Hinterbliebenenrenten für Witwen und Witwer werden durch einen rentenrechtlichen Beitragsausgleich für den Aufbau der eigenständigen Alterssicherung in der Partnerschaft abgelöst. Der mit dem Sicherungsbedarf von Erwerbsfähigen unvergleichbare Bedarf von Kindern bedingt, dass für den Fall des Todes Versicherter unverändert Waisen- und Erziehungsrenten gezahlt werden.

3. Beitragsfreie Zeiten, die bisher kleinteilig zum Zeitpunkt des Rentenbeginns erstmals bewertet werden und für die im Zeitverlauf Art und Umfang der Berücksichtigung mehrfach verändert wurde, werden mit dem Grundbetrag berücksichtigt und damit prospektiv kalkulierbar.

Die mit diesen drei Elementen berechnete Rente soll wie bisher im Umlageverfahren aus den Beiträgen der Versicherten und ihrer Arbeitgeber finanziert werden. Hinzu kommt der Bundeszuschuss, der im gesamtgesellschaftlichen Interesse liegende Leistungen wie die Integration der neuen Bundesländer und Elemente des sozialen Ausgleichs für die Rentenversicherung finanziert.

Der steuerfinanzierte Grundbetrag soll in bis zu 30 Jahren anteilig entsprechend der Versichertenzeit erworben werden. Mindestbeitragszeit sind fünf Jahre. Mit 30 Jahren ist der volle beitragsunabhängige Grundbetrag angesammelt. Der Sockel wird nach Entgeltpunkten bestimmt, die mit dem allgemeinen Rentenwert angepasst an die Einkommensentwicklung bewertet werden. Der allgemeine Rentenwert ist seit dem 1. Juli 2009 für die neuen Bundesländer auf 24,13 Euro und für die alten Bundesländer auf 27,20 Euro festgesetzt. Die Höhe des Sockels soll nach 30 Jahren unter der Mindestsicherung des SGB XII liegen. Für die jeweilige individuelle Rente kommt zum Grundbetrag der beitragsabhängige Steigerungsbetrag hinzu. Er ist abhängig von der Zahl und der Höhe der Entgeltpunkte und wird nach dem Äquivalenzprinzip bestimmt. Wie im geltenden Recht ${ }^{2}$ wird der Steigerungsbetrag aus der Multiplikation der individuellen Entgeltpunkte mit dem aktuellen Rentenwert errechnet. Hier liegt der Leistungsanreiz. Die drei Jahre Kindererziehungszeiten je Kind werden unverändert als Entgeltpunkte im Sockel rentenbegründend und im Steigerungsbetrag rentensteigernd angerechnet.

Die Rente mit Grundbeitrag ist qualitativ mehr als eine bedürftigkeitsabhängige Grundsicherung im Alter, wie sie die Sozialhilfe des SGB XII vorsieht. Die steuerfinanzierte Grund-

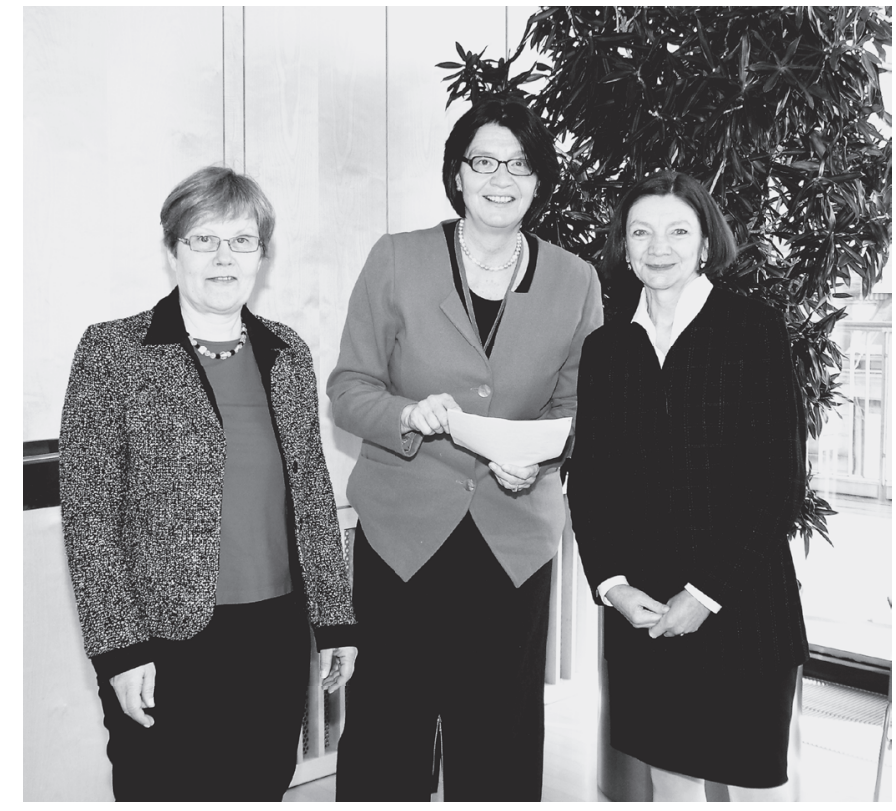

A Das Thema Rente gilt als kompliziert: Prof. Dr. Ursula Rust, Universität Bremen (links) sprach über die Rentenreform 2007 und die mittelbare Diskriminierung der Frauen durch die Rente für besonders langjährige Versicherte (45 Beitragsjahre), neben ihr Christel Riedel (Mitte) und die Präsidentin des djb Jutta Wagner.

sicherung im Alter nach dem SGB XII verzichtet zwar - anders als früher - auf den Rückgriff bei den Kindern. Es ist aber unverändert eine steuerfinanzierte Sicherung im Alter, die bedürftigkeitsgeprüft mit allen daraus folgenden sozialrechtlichen Pflichten ist. Der Verzicht auf den Rückgriff auf Kinder ist ein wichtiger Schritt gewesen, um verschämte Altersarmut zu vermeiden. Dies ist aber nicht genug. Im Alter ist die beitragsfinanzierte gesetzliche Altersversorgung die wichtigste Einkommensquelle ${ }^{3}$. Hier ist die eigenständige, armutsvermeidende Altersvorsorge für Frauen zu verankern.

Innerhalb einer bestehenden Ehe soll nach dem Modell das Einkommen den Beitragskonten der beiden Ehepartner jeweils getrennt in Höhe der Hälfte des Gesamteinkommens zugeschrieben werden. Witwen- und Witwerrenten werden damit von einem rentenrechtlichen Beitragsausgleich abgelöst. Der rentenrechtliche Ausgleich findet nur innerhalb einer bestehenden Ehe statt und ersetzt den jetzigen Versorgungsausgleich. Der rentenrechtliche Beitragsausgleich endet mit Auflösung der Ehe. Sofern ein Ehegatte vor Erreichen der Altersgrenze stirbt, wird die damit entstehende Beitragslücke wie bisher mit der Erziehungsrente aufgefangen.

1 www.djb.de/Kommissionen/kommission-recht-der-sozialensicherung-familienlastenausgleich/djb-modell/ und aktuelle Informationen des djb Nr. 1/2004, S. 24-27.

2 S. Beitrag Henze, „Beitragsäquivalente Alterssicherung“, S. 108 f. in diesem Heft.

3 S. ebd. 


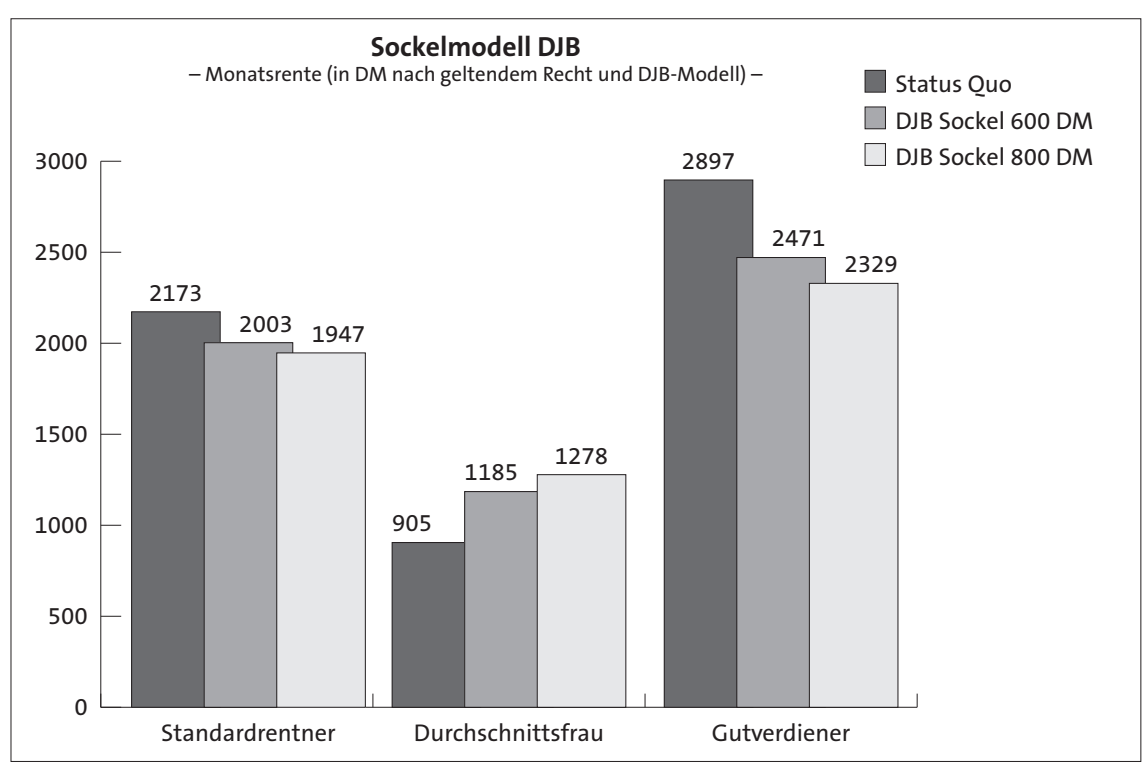

Gesetzentwurf zur Anpassung der Regelaltersgrenze an die demografische Entwicklung und zur Stärkung der Finanzierungsgrundlagen der gesetzlichen Rentenversicherung der Bundesregierung hat der djb auch $^{9}$ anlässlich der öffentlichen Anhörung im Bundestagsausschuss für Arbeit und Sozialordnung die Einführung der neuen Rentenart für besonders langjährig Versicherte darauf hingewiesen, dass von dieser neuen Rentenart Frauen mittelbar ausgeschlossen sind, da sie fast nie deren Voraussetzungen erfüllen. ${ }^{10}$ Bei der Anhörung hat der djb zwar nicht von den Parlamentariern, jedoch von anderen Sachverständigen $\mathrm{Zu}$ stimmung erhalten. Mit der Rente für besonders langjährig Versicherte ist erstmalig eine mittelbar geschlechtsdiskriminierende Vorschrift im Rentenrecht eingeführt wor-

In den Grundbetrag geht die bisherige Anrechnung von beitragsfreien Zeiten ein. Zeiten, die im allgemeinen Interesse auch ohne Beiträge für die Rente anzurechnen sind, werden nach dem Modell mit dem Grundbetrag pauschal abgegolten. Sie werden damit - anders als derzeit - nicht je besser bewertet, je höher der Verdienst und je kontinuierlicher die Beitragszahlung der Versicherten ist. Sie sind ein Element des sozialen Ausgleichs, der in den Grundbetrag und damit die rentenrechtliche Mindestsicherung einfließt.

Nach der Berechnung der Bundesversicherungsanstalt von 2000 zum Modell ist davon auszugehen, dass das gewünschte Ergebnis der maßvollen Umverteilung im System zugunsten von Frauen mit der Kombination von Grund- und Steigerungsbetrag erreicht wird. Die Berechnung von 2000 hat noch nicht den rentenrechtlichen Beitragsausgleich zwischen Partnern berücksichtigt. Ihr noch in DM gerechnetes Ergebnis wird gleichwohl hier mit dem Schaubild ${ }^{4}$ wiedergegeben, um die Verteilungswirkung zu veranschaulichen.

Mit der Rentenreform 2000 hat nach der ersten Vorlage des djb-Rentenmodells der Paradigmenwechsel für die Altersvorsorge stattgefunden, dass die gesetzliche Rentenversicherung durch die kapitalgestützte Eigenvorsorge zu ergänzen ist. Sie wird derzeit steuerlich gefördert. Das djb-Modell ist kurz vor diesem Systemwechsel in der Kommission Soziale Sicherheit in dem Umfang konkretisiert worden, dass es bei einer Fachkonferenz 2000 mit rentenrechtlichen Expertinnen und Experten kritisch diskutiert werden konnte. Es ist seitdem ein Konzept, auf das in der wissenschaftlichen Literatur immer wieder hingewiesen wird ${ }^{5}$. Die Kommission hat das Modell in der Mitgliederversammlung 2001 vorgestellt. Sie hatte Gelegenheit, umfassend zu der „Paradigmenreform“ Stellung zu nehmen. ${ }^{6}$ Ihre Forderung zur Einführung der zusätzlichen Eigenvorsorge, für die private Altersvorsorge zumindest UnisexTarife einzuführen ${ }^{7}$, ist 2000 nicht aufgegriffen worden. Die Forderung ist aber 2004 mit dem Alterseinkünftegesetz - wie vom djb vorgeschlagen - geltendes Recht geworden. ${ }^{8}$ Zum den, nachdem in den 1980er Jahren sämtliche Formen einer unmittelbar unterschiedlichen Behandlung wegen des Geschlechts abgeschafft worden waren. Dies Ärgernis darf aber nicht den Blick darauf verstellen, dass es für die Baustelle des geschlechtergerechten Umbaus der gesetzlichen Altersversicherung unverändert Handlungsbedarf gibt. Dies wird die Aufgabe für die nächste und kommende Kommissionen sein. Zum geschlechtergerechten Umbau der gesetzlichen Rentenversicherung gehört auch, die Kindererziehungszeiten von drei Jahren um die vor 1992 geborenen Kinder zu erweitern. Die AVID-Studie 2005 zeigt, dass bei bis zu zwei Kindern mit den Kindererziehungszeiten eine signifikante Angleichung der Alterseinkünfte von kinderlosen und Frauen mit Kindern erfolgt. ${ }^{11}$ Ein weiteres Eckdatum des djb-Konzepts ist die anstehende Erweiterung des versicherten Personenkreises hinsichtlich der Selbstständigen. Die Freistellung der Selbstständigkeit von der Verpflichtung zur Vorsorge für das Alter ist ein Risiko, das nicht mehr zu rechtfertigen ist, wenn damit Lücken entstehen, für die im Alter zunehmend die Grundsicherungsleistung des SGB XII einstehen müssen.

Prof. Dr. Ursula Rust, Mitglied der Kommission Recht der sozialen Sicherung, Familienlastenausgleich des djb; Universität Bremen

4 Thiede, ZSR 2000, 871.

5 Zuletzt von Reinhold Thiede in seinem Beitrag „Mindestsicherungselemente in der gesetzlichen Rentenversicherung?" in: WSI-Mitteilungen $07 / 2009$.

6 Tätigkeitsbericht 1999 bis 2001 mit Nachweisen.

7 Pressemitteilung 01-01.

8 Aktuelle Informationen 1/2004, 22-24, 41-42 und verschiedene Pressemitteilungen.

9 Pressemitteilungen $06-02$ und 07-24.

10 S. Beitrag Riedel, ,Ist Altersarmut unser (Frauen-)Schicksal?“, S. 110 ff. in diesem Heft.

11 Ebd. S. 111. 